STRUCTURAL SCIENCE CRYSTAL ENGINEERING MATERIALS

ISSN 2052-5206

\section{Bonding, Structure \& Solid-state Chemistry. By Mark Ladd. Oxford University Press, 2016. Pp. 527. GBP 32.50 (paperback), GBP 65.00 (hardback). ISBN 978-0-19- 872995-2}

\author{
Ulrich Müller* \\ Fachbereich Chemie, Philipps-Universität, D-35032 Marburg, Germany. *Correspondence e-mail: \\ mueller@chemie.uni-marburg.de
}

Keywords: book review; bonding; solid-state structures.

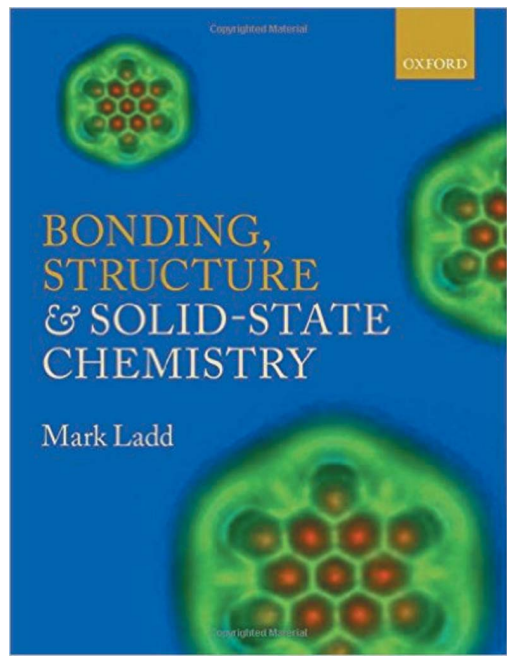

According to the author, the book 'should find its place in any year of the degree studies in the physical and biochemical sciences' and it 'presumes a background in science and mathematics of approximately A-level standard'. In fact, some more than basic knowledge of chemistry, physics and mathematics is required, such as integral calculus, symmetry notations and thermodynamics (free energy, entropy, enthalpy etc.). Also, many rather special terms are expected to be known, such as 'Fourier space', 'dimension of an irreducible representation' and 'relativistic contraction'.

Quantum theory is explained from scratch in Chapter 2 (117 pages), beginning with black-body radiation, Planck's theory, the De Broglie equation and Heisenberg's principle and then becoming quite elaborate with wavefunctions. This is well understandable, provided the reader is well grounded in mathematics. It continues elaborately with molecular orbitals, including delocalized $\pi$-bonds, multicentre bonds, ligand-field theory, character tables (without explaining the meaning of the character symbols) and briefly with VSEPR theory. Less satisfactory is that explanations are given quite a few times using terms that have not yet been introduced. This is a general didactic deficiency throughout the book. For example, knowledge of the terms $p$ electron and $\pi$-bonding is required to understand Example 2.6 on p. 47, but the terms are only explained later on pages $70 \mathrm{ff}$. In this case, the mentioned terms can be expected to be known to the reader in the first place, so there is no point in explaining them at all.

Chapter 3 (68 pages), 'Molecular compounds', begins with polarization, dipole interactions and intermolecular potentials, again with lots of mathematical formulae, including very briefly some related physical properties like the refractive index. There is a superfluous section on 'vibrational bonding'. After a short discussion of the structure of liquids follows Section 3.12 on the structures of molecular solids. This is restricted to a small, unsystematic and arbitrary selection of a few solid-state structures; organic compounds are essentially presented by overcrowded figures and a superfluous table of bond lengths. The reader is referred to two structure databases, leaving unmentioned the Inorganic Structural Database and Pearson's Crystal Data. The reader is left at a loss with this Section 3.12; it should have been deleted.

Chapter 4 (62 pages) is dedicated to ionic compounds, with a main focus on crystal energetics and related topics such as electron affinities, solubilities, ionic radii and heat capacities. The alkali halide and $M X_{2}$ structures and their relations to ionic radius ratios are worked out clearly (although in Fig. 4.14 the alleged relationship between the $\mathrm{TiO}_{2}$ and $\mathrm{NaCl}$ type is not perceivable). A section on silicate structures points out their main structural principles, but not going into detail (e.g. the chrysotile structure is mentioned, but not that its silicate sheets are curled to tubes); nothing more recent than W. L. Bragg (1933) is cited (e.g. Libau's systematic book on silicates). Point defects, doping and electrical properties are subjects, including an explanation of how image plates work.

Chapter 5 (65 pages), 'metallic compounds', is something for physicists, hardly understandable for chemists. It is focused on physical properties and bonding theory. In parts (e.g. wave-mechanical free-electron theory, p. 291), the reader will have a hard time understanding much unless he or she already knows what is being explained. There are more obscurities, for example: 'species such as $\mathrm{Ti}^{3+}$ should be a better conductor than...' (p. 289); is $\mathrm{Ti}^{3+}$ a single ion or a solid or what? The chapter also deals with semi- 
conductors, superconductors, fuel cells, batteries and solar cells, with restriction to a few examples. The section on the structures of metals mentions only the three main packings of spheres and the section on alloys is restricted to copper-gold, silver-cadmium and the Hume-Rothery rules. High-pressure structures are not to be found.

Chapter 6 (30 pages) is concerned with nanostructures, especially with their physical properties. The main attention concerns different nano species of carbon. The preparation of nanomaterials is touched on briefly.

Chapter 7 (14 pages), 'computer-aided chemistry', describes several computer programs, mainly for the calculation and plotting of orbitals. I was not able to check these programs, because I could not find out how to access them at the mentioned publisher's website.

84 pages of appendices explain crystallographic relations, matrix and related calculus, other mathematical procedures, Boltzmann and quantum statistics, and a number of physical laws. This relieves the main chapters, but can be annoying when the reader is forced to swap between the main part and the appendices.

Generally, the artwork is good, but not throughout. The curves in Figs. 2.7 and 2.8 are not accurate. Nothing can be recognized in the unnumbered figure on p. 200. In Fig. 3.29 the alleged close-packing of $\mathrm{C}_{2} \mathrm{Cl}_{6}$ is not discernible. Many figures of crystal structures are confusing because of overcrowding, even though they are depicted as stereoviews (pp. 207-210, $247,250)$. In Fig. 5.5 it is not clear why point $k$ should be labelled $\overline{1} 11$. Depicting less in Fig. 6.15 would show more.

Some confusion is created in formulae by the multiple use of the same symbol with different meanings, and the list of notations in the front matter is pretty incomplete. For example, $\alpha$ means polarizability or the coefficient of thermal expansivity according to the list of notations, but is also used for the angle of scatter, crystallographic interaxial angle, irreducible representation and certain variables in molecular orbital calculations. $V$ sometimes means volume, sometimes potential energy, which sometimes is also named $U$. It is not explained why a term of $5 / 2 R T$ is included in the lattice energy calculations.

Here follows a short selection of inaccuracies, inconsistencies and linguistic lapses: What is a 'six-coordinated solid' (p. 2)? In Section 1.6.2 it is said that a gradual (second order) transition may exhibit hysteresis; that is not correct. The equation for the $C_{2}$ symmetry operation on p. 91 is unsolvable. According to p. 91, 'the trace, or character, of a matrix is the sum of the diagonal elements', but on p. 92 the character of $E$ (identity) is given as 1 while the trace of its matrix is 3. The formula of $\mathrm{SO}_{2}$ on p. 114 is wrong. Flat molecules allegedly have their planes nearly parallel in the solid state (p. 206), which very often is not true. The formula 4.2 is wrong (p. 216). Can a ring, chain or network be tetrahedral (p. 249)? Energies are given on p. 256 in kcal, on p. 258 in $\mathrm{kJ}$. Solid caesium chloride is said to contain only two ions ( $\mathrm{p}$. 260). A vacancy and a hole mechanism are distinguished on $p$. 267 , but what is the difference between a vacancy and a hole? A 'monoatomic solid' is a solid that consists of merely one atom (p. 286)? What is a 'two-dimensional crystal'? (p. 358; crystals are always three-dimensional). What is 'a pentagonal graphene' (p. 359)?

There are only a few typographic errors, e.g. equations 2.144 and 2.145 (repeatedly $y$ instead of $x$ ) and the formula of $\mathrm{NH}_{3}$ (p. 115). It is disturbing that page column titles have no numbers and that the complete text does not contain any page references.

There is no real target-reading public for this book. It could be of interest for students of chemistry and physics, if they are not beginners. But then, much of the contents will be familiar to them, and beyond that, the treatment is often incomplete, too scarce and imprecise. The chapter on wavefunctions and molecular orbitals may give them new insights, unless they want to go deeper. I have the impression that the author, being retired, has found a pastime writing new books by shuffling the contents of his numerous previous books, without presenting anything new or beneficial. 